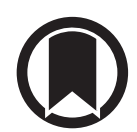

CrossMark

\title{
Adolescents and young adults: a neglected population group for tuberculosis surveillance
}

\author{
Alberto L. García-Basteiro ${ }^{1,2,3}$, H. Simon Schaaf ${ }^{4}$, Roland Diel $\mathbb{1}^{5}$ and \\ Giovanni Battista Migliori $\mathbb{1}^{6}$
}

Affiliations: ${ }^{1}$ Centro de Investigação em Saude de Manhiça (CISM), Maputo, Mozambique. ${ }^{2}$ ISGlobal, Barcelona Ctr. Int. Health Res. (ĆRESIB), Hospital Clínic - Universitat de Barcelona, Barcelona, Spain. ${ }^{3}$ Amsterdam Institute for Global Health and Development (AIGHD), Academic Medical Center, Amsterdam, The Netherlands. ${ }^{4}$ Desmond Tutu TB Centre, Dept of Paediatrics and Child Health, Faculty of Medicine and Health Sciences, Stellenbosch University, Cape Town, South Africa. ${ }^{5}$ Institute for Epidemiology, University Medical Hospital Schleswig-Holstein, Airway Research Center North (ARCN), Kiel, Germany. ${ }^{6}$ WHO Collaborating Centre for TB and Lung Diseases, Maugeri Care and Research Institute, Tradate, Italy.

Correspondence: Alberto L. García-Basteiro, Centro de Investigação em Saúde de Manhiça (CISM), Rua 12 Cambeve, Manhiça, Maputo 1929, Mozambique. E-mail: alberto.garcia-basteiroßmanhica.net

@ERSpublications

Improved surveillance systems are essential for understanding the true burden of TB in adolescents and young adults http://ow.ly/94Pz30iaixA

Cite this article as: García-Basteiro AL, Schaaf HS, Diel R, et al. Adolescents and young adults: a neglected population group for tuberculosis surveillance. Eur Respir J 2018; 51: 1800176 [https://doi.org/10.1183/ 13993003.00176-2018].

Tuberculosis (TB) remains a serious public health concern and it is considered the leading infectious cause of death globally. The World Health Organization (WHO) Global Tuberculosis Report of 2017 showed an estimated 10.4 million new TB cases for 2016, of which 6.7 million (64.4\%) were males and 1.04 million (10\%) were children $0-14$ years of age [1]. Unfortunately, these figures represent estimates, an attempt to quantify the real number of $\mathrm{TB}$ cases, since the notifications provided from many national surveillance systems, including some of the high-burden TB countries, fail to capture many TB cases. This is either because TB cases are not diagnosed or not recorded and reported to the health authorities. In fact, the WHO estimates that only $61 \%$ of all TB cases are notified to national health authorities [1]. Other available global estimates using a different methodology, such as those by the Institute for Health Metrics and Evaluation (IHME), derived from the Global Burden of Disease project, present a similar diagnostic gap of at least one-third of the cases [2]. Trying to close this gap between real and diagnosed/reported cases (those who are likely to receive adequate treatment) with active case-finding strategies has been the target of many TB control efforts, with different levels of success [2]. The premise is clear: undiagnosed cases have much higher mortality and perpetuate the spread of the disease within communities $[3,4]$.

The gap between the real number of cases and reported cases is probably not the same for all age groups. Underdiagnosis of TB is probably higher in children than in adults $[1,5]$. There are several reasons that TB diagnosis is more challenging in children. First, obtaining suitable respiratory secretion samples for microbiological confirmation of TB is difficult. Young children do not spontaneously expectorate sputum, and induced sputum and gastric aspirate collections are rarely performed [6]. Secondly, microbiological tests have reduced sensitivity in children's samples, because of the paucibacillary nature of TB in children [7].

Received: Jan 262018 | Accepted: Feb 012018

Conflict of interest: None declared.

Copyright OERS 2018 
Thirdly, symptoms, especially in young children, are often acute and non-specific, and their TB is commonly associated with other respiratory infections on presentation, leading to misdiagnosis and incorrect management [8]. Lastly, the pathogenesis and clinical presentation of TB in children are different from that in adults, reducing the sensitivity and specificity of adjunctive diagnostic tools, such as conventional radiology, which, on the other hand, is often unavailable or of poor quality in low income countries [8,9]. Child TB cases are therefore often missed and microbiological confirmation is low [10], leading to some uncertainty about the quality of TB diagnosis in notified children. Furthermore, reporting of child TB cases to national TB programmes has been suboptimal [11, 12]. Many countries do not disaggregate paediatric TB cases by smaller age groups, sex or microbiological confirmation, and until recently, some countries only reported microbiologically confirmed cases [13]. In countries where the private sector plays an important role in health delivery, the likelihood of missed notifications despite correct diagnosis is potentially higher [14]. Although paediatric TB has been historically neglected, there has been a renewed interest and awareness of this public health problem in recent years, which has led to efforts to improve its surveillance and reporting, as well as to develop new therapeutic formulations and, to a lesser extent, new diagnostic tests [5, 15-17].

Older children (5-10 years), adolescents (10-18 years) and young adults (19-24 years)[18] have been reported to have lower TB incidence rates than older adults (>25 years) (figure 1) $[19,20]$. Interestingly, in adolescents higher incidence has been reported for females than for males, especially in high incidence countries, which is an exception to the general TB profile in other age groups (higher rates in males) [21]. Adolescents and young adults are more likely to be TB infected and have a higher risk of being infectious than children do. Whether older adolescents have a higher risk of infection than younger ones is not entirely clear and probably depends on the intensity of transmission and overall TB prevalence (setting-specific) [22-24]. Nonetheless, it is clear that in adolescence and young adulthood TB notification rate increases steadily. Based on this data, TB researchers have concluded that a potential new TB vaccine offering protection for at least a few years against active $\mathrm{TB}$, would have higher impact in the short term if vaccination is administered in adolescents rather than in infants [25]. In addition, the size of this age group globally is considerable (almost 2 billion) and about nine out of 10 people between the ages of 10 and 24 years live in low income countries [18], which also often have the highest burden of TB.

Good surveillance systems, capable of detecting most TB cases occurring in communities, are essential for understanding the true burden and specific features of TB in adolescents and young adults. The different sociological, biological and behavioural patterns of adolescents and young adults model the TB epidemic, but little attention has been given to understanding the burden and drivers of TB in this population subgroup. The lack of individualised reporting data from countries with highest TB rates and the focus on the classical cut-off of under or above 15 years of age hinders the understanding of the real contribution of adolescents and young adults to the overall TB burden. Moreover, having good quality data on TB burden among adolescents and young adults will contribute to the design of vaccine studies with TB disease and TB infection efficacy end-points [26].

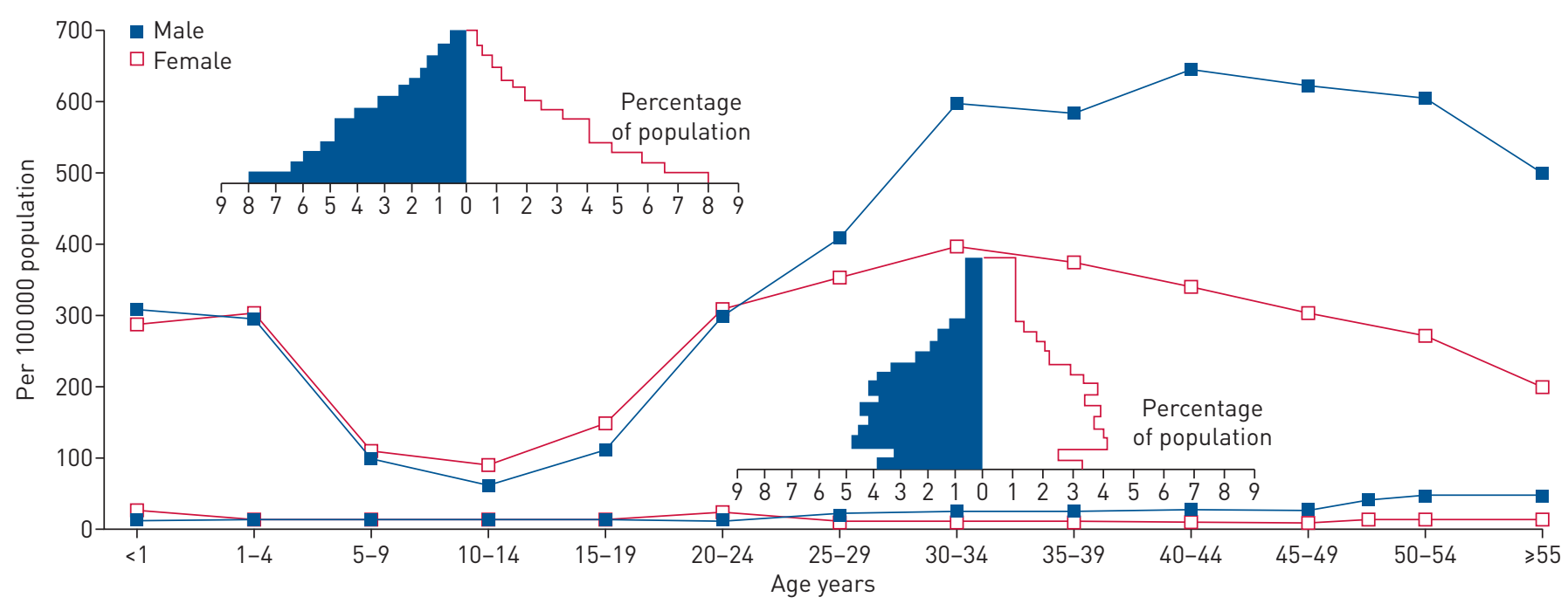

FIGURE 1 The age- and gender-related incidence of tuberculosis in a hypothetical high tuberculosis incidence community with a large number of children and a low tuberculosis community with a relatively small number of children. Reprinted with permission of the International Union Against Tuberculosis and Lung Disease [19]. Copyright @T The Union. 
In this issue of the European Respiratory Journal, SNOw et al. [27] estimated a global number of incident TB cases among adolescents and young adults (10-24 years) of 1.78 million in 2012, representing $17 \%$ of all new cases. Importantly, they show an upward trend in the TB caseload from 10 to 24 years of age, which could partially be attributed, as the authors acknowledge, to increasing rates of HIV. However, similar patterns in low HIV, high TB burden areas (i.e. South East Asia), could support the idea of increasing risk of $\mathrm{TB}$ as young adolescents grow older. The estimates of the proportions of sputum smear-positive, sputum smear-negative and extrapulmonary TB cases by age group and country certainly adds relevant information, which can be used for the implementation of specific preventive strategies. An attempt to stratify by HIV status in this analysis would have been also very relevant in order to see the magnitude of the different risks of TB among HIV-infected and-uninfected people. Nonetheless, this effort represents a step forward in our understanding of the burden of TB in a completely neglected group within overall TB control and surveillance efforts.

As mentioned, the lack of stratified reporting data for many countries hinders the complex undertaking of obtaining the best estimates of the true number of cases. We believe that assumptions used by SNow et al. [27] and the use of data from several countries with different epidemiological profiles have contributed to overcome the lack of age-disaggregated data. However, the variability of TB case detection across countries entails high complexity and potential sources of error. This is clearly due to the data sources, assumptions and approaches used. The approach to case detection rate calculation used by this report and by the WHO in general is a subject of controversy. For many countries, generally those with higher resources but lower burden of TB, TB cases are estimated using vital registration systems and are adjusted by a standard factor [28]. For other countries, the case detection rate is estimated based on notifications and prevalence survey data [29]. Few countries use data from capture-recapture analyses or from inventory studies [28]. However, for more than 70 countries, many of them with high TB burden, case detection rates were based on expert opinion [28], whose level of accuracy is uncertain. In fact, prevalence surveys in many cases have considerably modified the estimates based on this previous approach, both upwards or downwards (figure 2) [28]. Nonetheless, it is reassuring that a different modelling strategy used by the IHME for estimating global TB incidence shows similar results, both for all the populations, as well as for adolescents and young adults [30]. The differences observed in other TB burden indicators, such as mortality, do deserve further study.

Undoubtedly, new efforts on estimating burden of disease are always welcome. However, the gaps of information in adolescents and young adults remain enormous. We do not fully understand the magnitude and drivers of the increasing rates among HIV-negative adolescents and young adults, or
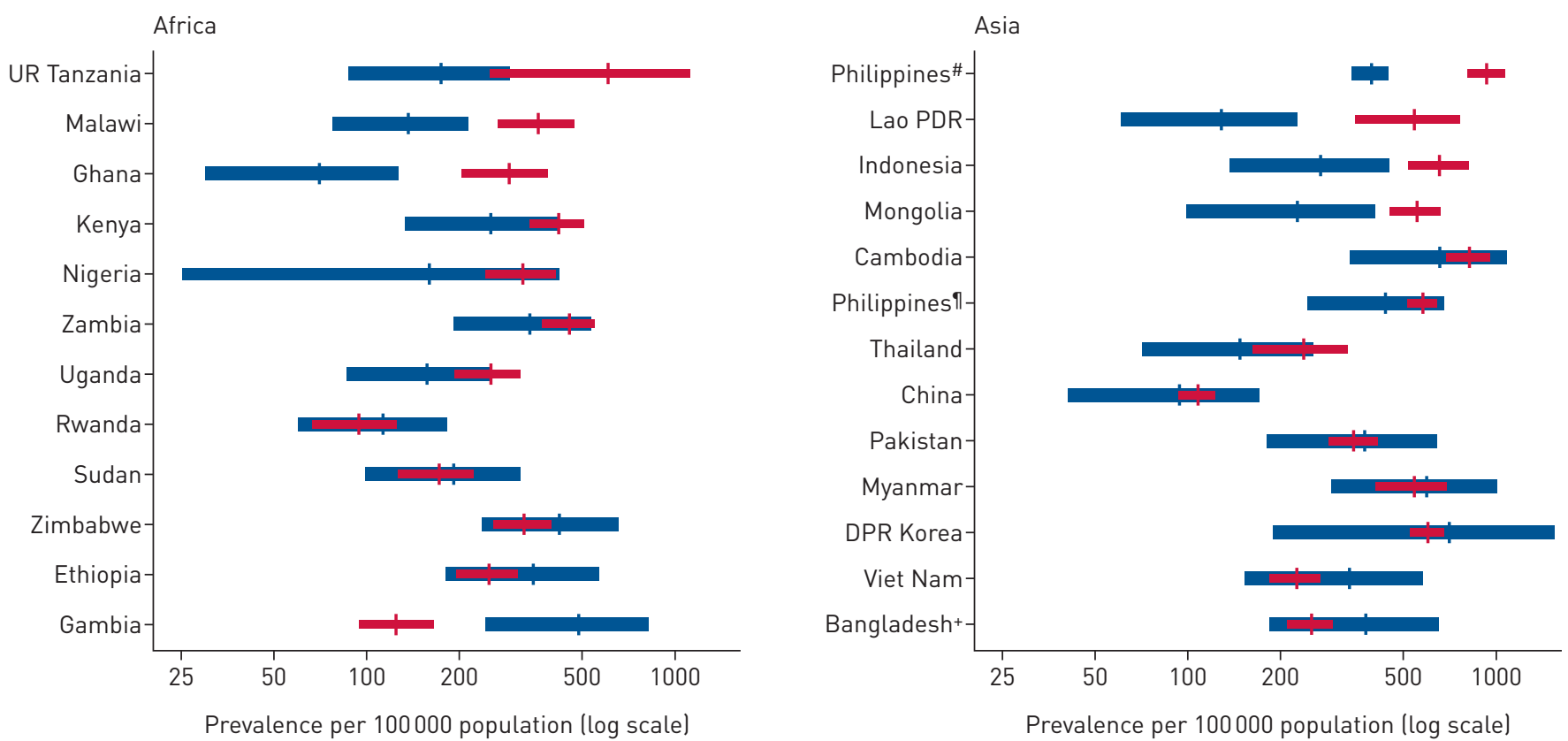

FIGURE 2 Estimates of TB prevalence (all ages, all forms of TB) for 25 countries, before (in blue) and after (in red) results from national TB prevalence surveys became available since 2007. Reprinted from the Global Tuberculosis Report 2017 [1] with permission of the World Health Organization. \#: these data relate to the repeat prevalence survey conducted in 2016 ; ${ }^{\text {" }}$ : these data relate to the prevalence survey conducted in $2007 ;^{+}$: these data relate to the prevalence survey conducted in 2015-2016. 
whether the apparent differences in TB notifications by sex in this population subgroup are real, and the reasons for these differences. A similar age-stratified prevalence of latent TB infection, as well as understanding the specific drivers of annual risk of infection and progression to disease in this population would be of huge value for TB vaccine development and, in general, for designing improved TB control strategies that can contribute to bend the TB epidemic curve downwards at a much higher rate.

\section{References}

1 World Health Organization. Global Tuberculosis Report 2017, WHO/HTM/TB/2017.23. Geneva, World Health Organization, 2017.

2 Blok L, Sahu S, Creswell J, et al. Comparative meta-analysis of tuberculosis contact investigation interventions in eleven high burden countries. PLoS One 2015; 10: e0119822.

3 Hermans S, Horsburgh CR Jr, Wood R. A century of tuberculosis epidemiology in the northern and southern hemisphere: the differential impact of control interventions. PLoS One 2015; 10: e0135179.

4 Tiemersma EW, van der Werf MJ, Borgdorff MW, et al. Natural history of tuberculosis: duration and fatality of untreated pulmonary tuberculosis in HIV negative patients: a systematic review. PLoS One 2011; 6: e17601.

5 Dodd PJ, Gardiner E, Coghlan R, et al. Burden of childhood tuberculosis in 22 high-burden countries: a mathematical modelling study. Lancet Glob Heal 2014; 2: e453-e459.

6 Nicol MP, Zar HJ. New specimens and laboratory diagnostics for childhood pulmonary TB: progress and prospects. Paediatr Respir Rev 2012; 12: 16-21.

7 Lamb GS, Starke JR. Tuberculosis in infants and children. Microbiol Spectr 2017; 5: 10.1128/microbiolspec. TNMI7-0037-2016.

8 Perez-Velez CM, Marais BJ. Tuberculosis in children. N Engl J Med 2012; 367: 348-361.

9 Marais BJ, Tadolini M, Zignol M, et al. Paediatric tuberculosis in Europe: lessons from Denmark and inclusive strategies to consider. Eur Respir J 2014; 43: 678-684.

10 Lopez-Varela E, Augusto OJ, Guerra L, et al. Low paediatric tuberculosis case detection rate in Southern Mozambique. Eur Respir J 2016; 47: 1003-1005.

11 Du Preez K, Schaaf HS, Dunbar R, et al. Incomplete registration and reporting of culture-confirmed childhood tuberculosis diagnosed in hospital. Public Heal Action 2011; 1: 19-24.

12 Lestari T, Probandari A, Hurtig A-K, et al. High caseload of childhood tuberculosis in hospitals on Java Island, Indonesia: a cross sectional study. BMC Public Health 2011; 11: 784.

13 Seddon JA, Jenkins HE, Liu L, et al. Counting children with tuberculosis: why numbers matter. Int J Tuberc Lung Dis 2015; 19: Suppl. 1, 9-16.

14 Cowling K, Dandona R, Dandona L. Improving the estimation of the tuberculosis burden in India. Bull World Health Organ 2014; 92: 817-825.

15 Jenkins HE, Tolman AW, Yuen CM, et al. Incidence of multidrug-resistant tuberculosis disease in children: systematic review and global estimates. Lancet 2014; 6736: 1-8.

16 World Health Organization. New fixed-dose combinations for the treatment of TB in children. 2016. www.who. int/tb/FDC_Factsheet.pdf Date last accessed: January 18, 2018.

17 Gjøen JE, Jenum S, Sivakumaran D, et al. Novel transcriptional signatures for sputum-independent diagnostics of tuberculosis in children. Sci Rep 2017; 7: 5839.

18 UNFPA. The state of the world population 2014. The power of 1.8 billion. Adolescents, youth and the transformation of the future. 2014. www.unfpa.org/sites/default/files/pub-pdf/EN-SWOP14-Report_FINAL-web. pdf Date last accessed: January 15, 2017.

19 Donald PR. Childhood tuberculosis: the hidden epidemic. Int J Tuberc Lung Dis 2004; 8: 627-629.

20 García-Basteiro AL, Respeito D, Augusto OJ, et al. Poor tuberculosis treatment outcomes in Southern Mozambique (2011-2012). BMC Infect Dis 2016; 16: 214.

21 Donald PR, Marais BJ, Barry CE. Age and the epidemiology and pathogenesis of tuberculosis. Lancet 2010; 375 : $1852-1854$.

22 Seddon JA, Shingadia D. Epidemiology and disease burden of tuberculosis in children: a global perspective. Infect Drug Resist 2014; 7: 153-165.

23 Middelkoop K, Bekker L-G, Myer L, et al. Rates of tuberculosis transmission to children and adolescents in a community with a high prevalence of HIV infection among adults. Clin Infect Dis 2008; 47: 349-355.

24 Mumpe-Mwanja D, Verver S, Yeka A, et al. Prevalence and risk factors of latent tuberculosis among adolescents in rural Eastern Uganda. Afr Health Sci 2015; 15: 851-860.

25 Knight GM, Griffiths UK, Sumner T, et al. Impact and cost-effectiveness of new tuberculosis vaccines in low- and middle-income countries. Proc Natl Acad Sci USA 2014; 111: 15520-15525.

26 Evans TG, Churchyard GJ, Penn-Nicholson A, et al. Epidemiologic studies and novel clinical research approaches that impact TB vaccine development. Tuberculosis 2016; 99: S21-S25.

27 Snow KJ, Sismanidis C, Denholm J, et al. The incidence of tuberculosis among adolescents and young adults: a global estimate. Eur Respir J 2018; 51: 1702352.

28 World Health Organization. Global Tuberculosis Report 2016. Geneva, World Health Organization, 2016.

29 Diouani MF, Ouerghi O, Refai A, et al. Detection of ESAT-6 by a label free miniature immuno-electrochemical biosensor as a diagnostic tool for tuberculosis. Mater Sci Eng C 2017; 74: 465-470.

30 Global Burden of Disease, Tuberculosis Collaborators. The global burden of tuberculosis: results from the Global Burden of Disease Study 2015. Lancet Infect Dis 2017; in press [https://doi.org/10.1016/S1473-3099(17)30703-X]. 